\title{
THE INFLUENCE OF THE FUTSAL OUTFIELD GOALKEEPER ON PLAYERS' RUNNING PERFORMANCE
}

original paper

() Wroclaw University of Health and Sport Sciences

DOI: https://doi.org/10.5114/hm.2022.107977

\section{JAN-PAUL JELMER DE JONG ${ }^{1,2}$, FABIO GIULIANO CAETANO ${ }^{2 \oplus}$, LAURA MARIA SASKIA DE JONG ${ }^{3 \oplus}$, VITORPANULA DA SILVA ${ }^{2 \oplus}$, MURILO JOSÉ DE OLIVEIRA BUENO ${ }^{2 \oplus}$, PAULO ROBERTO PEREIRA SANTIAGO ${ }^{4}{ }^{\oplus}$, LUIZ HENRIQUE PALUCCI VIEIRA ${ }^{4 \oplus}$, FÁBIO YUZO NAKAMURA ${ }^{5 \oplus}$, FELIPE ARRUDA MOURA ${ }^{2 \oplus}$}

\footnotetext{
${ }^{1}$ University of Groningen, Groningen, the Netherlands

${ }^{2}$ State University of Londrina, Londrina, Brazil

${ }^{3}$ Deakin University, Geelong, Australia

${ }^{4}$ University of São Paulo, Ribeirão Preto, Brazil

${ }^{5}$ Federal University of Paraíba, João Pessoa, Brazil
}

\begin{abstract}
Purpose. This study aimed to investigate the influence of the outfield goalkeeper game situation on the running performance of teammates and opponent players during professional futsal matches.

Methods. The movement trajectories of 126 professional players during 6 official matches were analysed with a videobased tracking system $(30 \mathrm{~Hz})$. Distance covered and percentage of distance covered in different speed ranges were calculated to identify differences per team and per sub-phase of the game (traditional vs. outfield goalkeeper situation).

Results. The teammates of the outfield goalkeeper spent a higher percentage of the distance covered in the standing and walking speed range $(36.27 \pm 15.38 \%)$ compared with the teammates of the traditional goalkeeper $(31.44 \pm 7.74 \%)$. The outfield goalkeeper spent a smaller percentage of the distance covered in the standing and walking range $(41.13 \pm 10.86 \%$ vs. $80.77 \pm 4.30 \%)$ and a higher percentage in the low- and medium-intensity running range $(31.59 \pm 5.59 \%$ vs. $15.07 \pm$ $2.74 \%$ and $14.33 \pm 3.90 \%$ vs. $2.50 \pm 1.19 \%$, respectively), as well as covered a greater distance $(77.41 \pm 16.00 \mathrm{~m} / \mathrm{min})$ than the traditional goalkeeper $(33.81 \pm 3.66 \mathrm{~m} / \mathrm{min})$.
\end{abstract}

Conclusions. These findings provide coaches with a background concerning the requirements during the outfield goalkeeper situation as guidelines for final exercises in training sessions.

Key words: indoor football, game analysis, computational tracking, outfield player, goalkeeper, work rate

\section{Introduction}

Since the 1930s, the game of futsal has been developing into an internationally recognized sport with several competitions at the national and the international level. Nowadays, there are several video-based systems making it possible to collect different kinematic variables (i.e. position, speed, acceleration) of the match $[1,2]$. These tools allow to quantify variables related to running performance, such as distance covered, movement categories and corresponding speed thresholds, sprints, and sequences of repeated sprints [3, 4].

Several researchers who investigated these variables during futsal matches $[3,5,6]$ concluded that futsal was a multiple-sprint sport with high-intensity phases [7] in which players at a higher competitive level covered greater distances at a high speed $(>5 \mathrm{~m} / \mathrm{s})$ $[5,6]$. Additionally, it was found that during match-

Correspondence address: Felipe Arruda Moura, Laboratory of Applied Biomechanics, Department of Sport Sciences, Centre of Physical Education and Sport, State University of Londrina, Km 380 Celso Garcia Cid Road - University Campus, Londrina, Paraná, 86057-970, Brazil, e-mail: felipemoura@uel.br, https://orcid.org/0000-0002-0108-7246

Received: August 25, 2020

Accepted for publication: February 9, 2021

Citation: de Jong J-PJ, Caetano FG, de Jong LMS, da Silva V, de Oliveira Bueno MJ, Santiago PRP, Vieira LHP, Nakamura FY, Moura FA. The influence of the futsal outfield goalkeeper on players' running performance. Hum Mov. 2022;23(3):49-55; doi: https://doi.org/10.5114/hm.2022.107977. 


\section{HUMAN MOVEMENT}

J. de Jong et al., Futsal outfield goalkeeper

play, short repeated sprints (recovery $<15 \mathrm{~s}$ ) were more frequent than repeated sprints with longer recovery times in between, regardless of the playing position [3]. This emphasizes that futsal is a highly intense and dynamic sport, since players react to high-intensity runs of the opponents. With regard to the total distance covered during a match, literature reported smaller values in friendly matches than in official matches [8] and during the second match-half compared with the first one, even when only in-play time was considered [9]. Furthermore, the research shows an increase in sprint duration in the second half compared with the first half [3]. However, the aforementioned physical demands may change when a team chooses to play with an outfield goalkeeper (OGK).

OGK is an often-used tactic of the team at losing hand during the end of a match. According to the FIFA laws of the game [10], any teammate may substitute the goalkeeper and does not necessarily need to inform the referee or wait for a stoppage in the game. This tactic is often applied by the team at losing hand during ball possession to increase scoring opportunities [11]. When using an OGK, the attacking team will outnumber the defending team, which may lead to a different spatial organization for both the attacking and defending team [2]. Moreover, the sprint duration increases in the second half compared with the first half [3]. Up to date, it is unclear if this increase is a result of the goalkeeper substitution.

The findings that sprint duration increases in the second half and that in an OGK situation the positioning of players changes lead to the question if this change in running performance will arise specifically owing to the OGK situation. To the extent of our knowledge, this issue has only been addressed in tactical behaviour during training situations [2] or during official matches [12]. One can argue that players of the defending team need to cover greater distances at a higher speed than the attacking team because of the numerical inferiority. Additionally, if there is numerical superiority in terms of outfield players, it is expected that the attacking team may present a lower distance covered by the outfield players.

The purpose of this research was to characterize the running performance of the goalkeeper, the outfield players, and the opponent players in the OGK situation and to compare these demands with the traditional goalkeeper (TGK) situation in professional futsal matches. We hypothesized that in the OGK situation, the defending team would cover a greater distance and at higher intensities than the attacking team in comparison with the TGK situation. Furthermore, we expected higher requirements on running performance of OGK compared with TGK.

\section{Material and methods}

Participants and automatic tracking system

The automatic tracking system of the DVideo software [4] was used to obtain the trajectories of 126 professional futsal players, including goalkeepers. A total of 30 TGK (average duration: 29.5 minutes) and 24 OGK (average duration: 1.1 minutes) situations during 6 official matches of the Brazilian First Division Futsal League were analysed, which resulted in 150 and 144 teammates and opponents data samples for TGK situations, respectively, and 31 teammates and 37 opponents data samples for OGK situations. Overall, 6 official matches of the Brazilian First Division Futsal League were examined. The matches were recorded by 2 digital cameras $(30 \mathrm{~Hz})$, fixed at elevated positions in the gymnasium. Each camera covered approximately 3 quarters of the court, with an overlapping region between them. The images were transferred to a central computer and synchronized by identifying a common event in the overlapped region, such as a kick by a player. The players' $2 \mathrm{D}$ positions relative to the court as a function of time were obtained with an automatic tracking system. The average error of $9.8 \mathrm{~cm}$ and $0.8 \%$ was presented for the determination of player position and the distance covered, respectively [9]. The system specifications are available elsewhere $[3,9]$. Here, we used the same database as in the aforementioned companion papers to (re)analyse distinct aspects of futsal matches performance. Finally, the players' trajectories were filtered by using a third-order low-pass digital Butterworth filter with a cut-off frequency of $0.4 \mathrm{~Hz}$.

\section{Data analysis}

The distance covered by each player was calculated as the cumulative sum of the displacement between 2 consecutive frames. The total distance covered by each player was normalized for the minutes the player participated in the match [9]. In addition, the percentage of the distance covered was computed by using the following speed ranges [13]: $\mathrm{S} 1 \leq 6 \mathrm{~km} / \mathrm{h}$ (standing and walking); $6<\mathrm{S} 2 \leq 12 \mathrm{~km} / \mathrm{h}$ (low-intensity running); $12<\mathrm{S} 3 \leq 15.4 \mathrm{~km} / \mathrm{h}$ (medium-intensity running); $15.4<\mathrm{S} 4 \leq 18.3 \mathrm{~km} / \mathrm{h}$ (high-intensity running); 
and S5 $>18.3 \mathrm{~km} / \mathrm{h}$ (sprinting). Finally, the instants of time when the goalkeeper played as an outfield player were identified and the analyses were performed separately for the TGK and the OGK situations.

\section{Statistical analysis}

Prior to each analysis, the Lilliefors test was performed to identify if the data were normally distributed and Levene's test to establish the variance of the data. Because normal distribution or homogeneity of the variance was absent in all situations (except for the data of the distance covered by the goalkeepers), the Box-Cox transformation was performed to reduce anomalies such as non-additivity, non-normality, and heteroscedasticity. A two-way analysis of variance (twoway ANOVA) was used to compare the total distance covered by the players with reference to 2 factors: game situation (TGK and OGK situation) and team (opponents and teammates of TGK and OGK). The percentage of distance covered by outfield players was compared through a three-way ANOVA with reference to speed ranges (S1, S2, S3, S4, and S5), game situation (TGK and OGK situation), and team (opponents and teammates of TGK and OGK). The distance covered by goalkeepers was compared through an independent $t$-test (TGK and OGK). A one-way ANOVA was utilized to compare the percentage of distance covered by the goalkeepers in each speed range in accordance with the speed ranges (S1, S2, S3, S4, and S5). When differences were found, Tukey's post-hoc test was applied. A significance level of $\alpha=0.05$ was used for all statistical analyses. The effect sizes were calculated and classified as small $(d>0.20)$, medium $(d>0.50)$, and large $(d>0.80)$ [14]. The variables are expressed as means \pm standard deviations. All statistical procedures were performed with the MATLAB ${ }^{\circledR}$ software (2015a, MathWorks Inc., Natick, MA, USA).

\section{Ethical approval}

The research related to human use has complied with all the relevant national regulations and institutional policies, has followed the tenets of the Declaration of Helsinki, and has been approved by the Ethical Committee of the University of Londrina (protocol number: 02074712.0.0000.5231).

\section{Informed consent}

Informed consent has been obtained from all individuals included in this study.

\section{Results}

The relative distance covered by OGKs $(77.43 \pm$ $16.00 \mathrm{~m} / \mathrm{min}$ ) was significantly greater $(p<0.01 ; d=4.62$, large) than that covered by TGKs $(33.81 \pm 3.66 \mathrm{~m} / \mathrm{min})$. However, no significant difference in the distance covered by the outfield players $(\mathrm{F}(1,361)=0.17 ; p=0.68)$ was found (Figure 1). An example of goalkeepers' trajectories during approximately 2 minutes of a given match (stoppages included) and a representation of the players' positions in the TGK and OGK situations are shown in Figure 2.

As for the percentage of distance covered in the different speed ranges (Table 1), TGKs presented higher values in the S1 range. In contrast, OGKs showed a higher percentage of distance in the $\mathrm{S} 2$ and $\mathrm{S} 3$ ranges $(\mathrm{F}(4,89)=124.61 ; p<0.01 ; d=4.28-5.63$, large $)$.

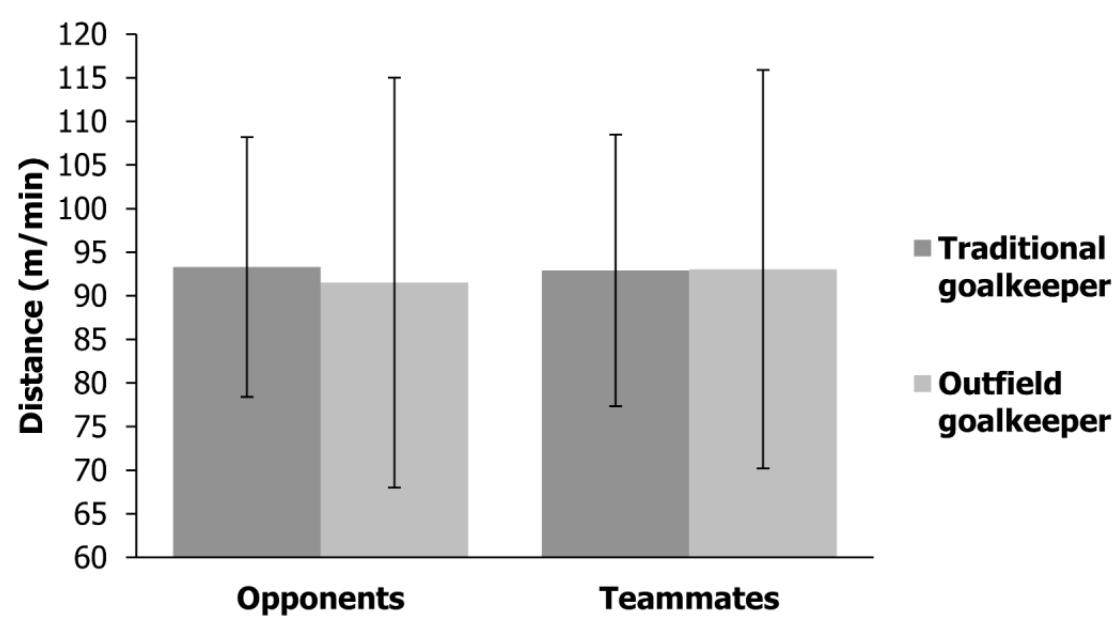

Figure 1. The mean distance of the teammates and opponents of the traditional and outfield goalkeeper 


\section{HUMAN MOVEMENT}

J. de Jong et al., Futsal outfield goalkeeper

A

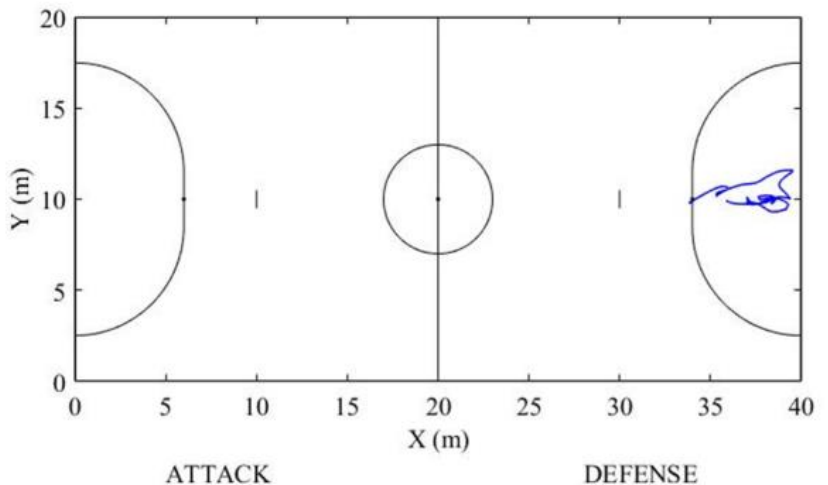

C

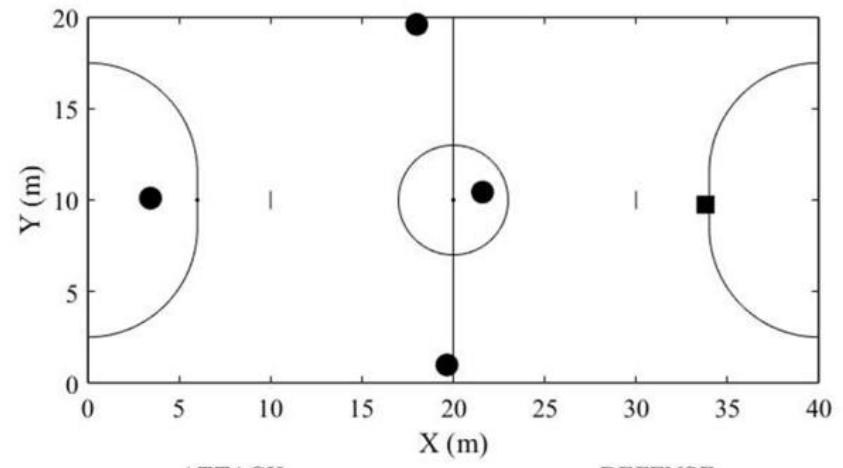

B

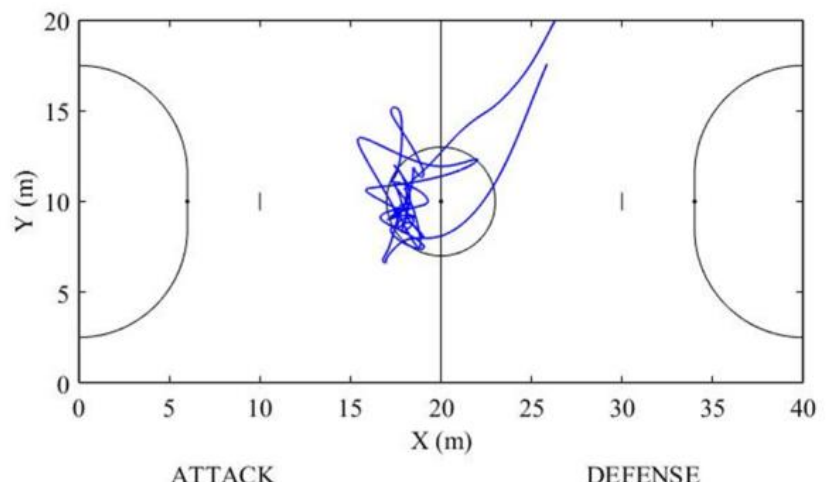

D

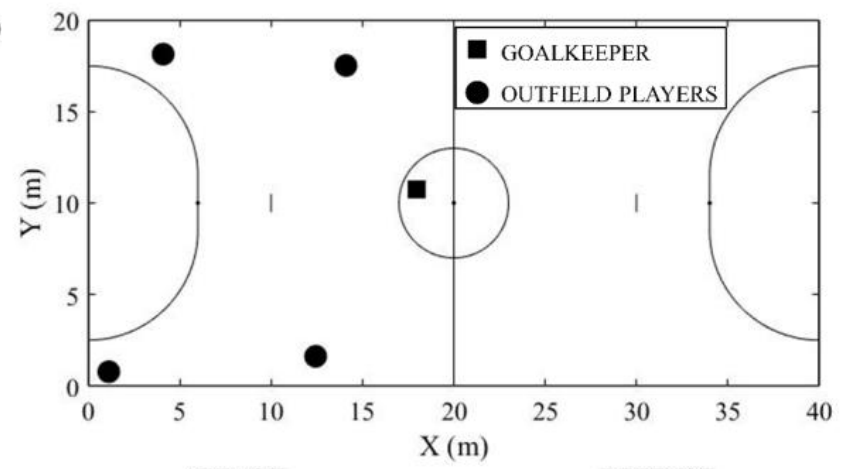

ATTACK

Figure 2. An example of the trajectory of the traditional goalkeeper (A) and the outfield goalkeeper (B). An example of the positions of outfield players (circles) and goalkeepers (squares) in the traditional goalkeeper situation (C) and the outfield goalkeeper situation (D)

Table 1. Percentage (mean $\pm S D$ ) of distance covered by goalkeepers in different speed ranges

\begin{tabular}{lcc}
\hline Speed range & $\begin{array}{c}\text { Traditional } \\
\text { goalkeeper } \\
\text { situation }\end{array}$ & $\begin{array}{c}\text { Outfield } \\
\text { goalkeeper } \\
\text { situation }\end{array}$ \\
\hline Standing and walking & $80.77 \pm 4.30^{*}$ & $41.13 \pm 10.86$ \\
Low-intensity running & $15.07 \pm 2.74$ & $31.59 \pm 5.59^{\#}$ \\
Medium-intensity running & $2.50 \pm 1.19$ & $14.33 \pm 3.90^{\#}$ \\
High-intensity running & $1.02 \pm 0.72$ & $6.45 \pm 3.29$ \\
Sprinting & $0.64 \pm 0.49$ & $6.50 \pm 5.06$ \\
\hline
\end{tabular}

* significantly higher $(p<0.01)$ for the traditional goalkeeper

\# significantly higher $(p<0.01)$ for the outfield goalkeeper
When comparing the percentage of distance covered by outfield players (opponents and teammates of TGK and OGK) in the different speed ranges (Table 2), a significant difference was only found for the teammates of OGK, who presented higher values as compared with the teammates of $\operatorname{TGK}(\mathrm{F}(4,1809)=2.22$; $p<0.01 ; d=0.51$, medium).

\section{Discussion}

The main purpose of this study was to investigate differences in the running performance between the TGK and OGK situations in futsal. The results showed

Table 2. Percentage (mean $\pm S D$ ) of distance covered by outfield players in different speed ranges

\begin{tabular}{lcccc}
\hline \multirow{2}{*}{ Speed range } & \multicolumn{2}{c}{ Traditional goalkeeper } & \multicolumn{2}{c}{ Outfield goalkeeper } \\
\cline { 2 - 5 } & Opponents & Teammates & Opponents & Teammates \\
\hline Standing and walking & $31.50 \pm 9.47$ & $31.44 \pm 7.74$ & $35.22 \pm 9.73$ & $36.27 \pm 15.38^{\#}$ \\
Low-intensity running & $39.44 \pm 6.30$ & $40.09 \pm 5.19$ & $42.84 \pm 6.44$ & $40.37 \pm 10.79$ \\
Medium-intensity running & $15.17 \pm 4.46$ & $14.54 \pm 4.09$ & $11.95 \pm 4.95$ & $14.38 \pm 7.16$ \\
High-intensity running & $7.10 \pm 2.55$ & $6.94 \pm 2.72$ & $4.85 \pm 3.39$ & $5.22 \pm 3.26$ \\
Sprinting & $6.78 \pm 3.86$ & $7.00 \pm 4.41$ & $5.14 \pm 6.15$ & $3.76 \pm 3.66$ \\
\hline
\end{tabular}

\footnotetext{
\# significantly different $(p<0.01)$ from the teammates of the traditional goalkeeper
} 
that OGK, in comparison with TGK, spent a smaller percentage of distance covered in the S1 range and a greater percentage of distance covered in the S2 and $\mathrm{S} 3$ ranges. OGK covered more than twice the relative distance $(\mathrm{m} / \mathrm{min}$ ) of TGK. Also, the teammates (attacking team) of OGK presented a higher percentage of distance covered in the $\mathrm{S} 1$ range (walking) compared with the teammates (attacking team) of TGK.

That OGKs covered greater distances and presented a smaller percentage of distances in S1 may be explained by the fact that OGK is mainly involved in offensive sequences, for which they most often enter the field around the half-way line and stay in that position to be able to be substituted as soon as possible after losing ball possession. Also, OGK needs to move similarly to an outfield player, to be part of the attacking play. These behaviours are clearly represented in Figures $2 \mathrm{~A}$ and $2 \mathrm{~B}$, which depict the different goalkeepers' trajectories, as well as their influences on team organization on the court. This may explain similarities between the speed percentages for OGK and those for the teammates of OGK in the OGK situation (rightmost column in Tables 1 and 2). Even though sprinting is not significantly different for OGK compared with TGK, there seems to be a clear difference $(0.64 \%$ vs. $6.50 \%)$. Part of this increased demand for sprinting from OGK may be because they need to get in and out of the court very quickly. So, for practical implications, OGK training focused on high-intensity running is needed. On the other hand, TGK often stays around the goalkeeper area, which may explain the lower highspeed running and total distance covered.

An interesting finding of this study, with a medium effect size, is the higher percentage of distance covered in the $\mathrm{S} 1$ range for the teammates (attacking team) of OGK compared with the teammates (attacking team) of TGK (Table 2). This observation could be assigned to the fact that in numerical superiority, the attacking players have less space per person (5 instead of 4 players in the attacking half) and, therefore, fewer opportunities to move at high speeds. Additionally, since there is no significant difference in the total distance covered by the teammates (Figure 1), spending more time in lower speed ranges may represent an increase in the recovery periods during the match for the teammates of OGK. The increase in distance covered in S1 can enhance the high-intensity efforts in association football because more time spent in S1 could indicate more recovery time between sprints. This increase in distance covered in S1 might also enhance high-intensity efforts in futsal [15] as futsal is a high-intensity sport in which repeated sprints with a shorter recovery time occur more often than repeated sprints with longer recovery $[3,13]$.

Another explanation for the finding that the teammates of OGK (attacking team) spend a greater percentage of distance in S1 may be related to the tactical structure that the players adopt with this strategy. Both the attacking and defending team may change their tactical plan to adapt to the OGK situation, as identified in Figures 2C and 2D. Owing to the numerical superiority, the attacking players play the ball around and stay more in one specific region of the court (which results in a higher percentage of distance in S1) in order to exhaust the opponents. On the other hand, in the OGK situation, the defending team tries to compensate for the numerical outfield player advantage of the attacking team. This might be done by using zone defence and seeking to reduce the spaces afforded to the attacking players in the region of the goal [2]. Travassos et al. [2] showed an increased synchrony between the defending team and the ball, but the opposite for the OGK team (attacking team). This indicates that if the attacking team uses OGK, the defending team switches from man-marking to a zonal defensive system. By zonal marking, defending players constantly move to protect their goal area by following the ball movement, instead of focusing on the movement of the opponent players $[2,12]$. Attacking players use a more static system to play the ball around and to find a weakness in the defence $[2,12]$. This situation probably increases the S1 percentages for attacking players. It is not clear if one of the teams or both teams adapt their tactical system to the OGK situation or what the causal relation is between the change in situation and the result that the teammates of OGK (attacking team) spend a greater percentage of distance in S1. To understand this difference, further research is needed that would investigate the causal relationships between the changes in running performance and the changes in tactical behaviour during an OGK situation. The vector coding technique used by Moura et al. [16] could be useful to examine which team is leading in an OGK situation, since the technique provides a clearer insight into which team is leading the behaviour of the opponent at any moment of time, helping to understand the changes in running performance.

The limitations of the study are mainly related to data acquisition. In indoor sports, the use of real-time technologies, like global positioning systems or local positioning measurement [1], would enable a larger dataset. This, however, requires a local antenna system, which comes at a high cost. Instead, an accurate and low-cost video-based system was used, which, in 
addition, increased the processing time substantially. Moreover, notational data regarding event-data, such as shots on target, pass length, or pass accuracy, would add more contextual information about the match dynamics changes with the participation of OGK. Furthermore, understanding what portion of the increase in the total number of sprints is caused by the TGK and OGK running to the substitution area would be beneficial. Unfortunately, this was beyond the scope of this study and should be investigated in further research. It is also necessary to examine the possible change of the attacking or defensive system from 1 vs. 1 to zonal in the OGK situation. An in-depth analysis of which team is possibly leading a change in the coordination patterns will give more insights into the change in running performance.

The OGK running performance is greater in the higher speed ranges during the match compared with TGK. This is important for strength and conditioning coaches in tailoring their training programs to real match situations in which OGK is needed. For example, an OGK situation at the end of a training session simulates a higher running performance of defending players, unlike a TGK situation. The recovery of the attacking team might be faster, and attacking players learn to adapt to tactical changes of the defensive side, which might be useful to create more goal scoring opportunities.

Furthermore, OGK may be chosen as a tactical strategy to reduce energy expenditure within the team and enhance recovery because of the longer time spent in lower speed ranges. One can argue that with an increased recovery time, the ability to perform more or longer sprints at the end of the match will increase. Finally, it could be important to simulate these match situations during training sessions to prepare the athletes to better cope with the subtle changes in physical match demands.

\section{Conclusions}

In conclusion, the implementation of OGK in futsal changes the running performance during the game, both for the goalkeepers and for the outfield players. OGK has a higher total distance covered and spends a greater percentage of time in higher speed ranges than TGK. The OGK situation also changes the running performance of the outfield players, but only in the attacking team (i.e. OGK teammates). No change in running performance was found for the defending team, which may be due to a modification in the defensive system. These observations are useful for coaches and strength and conditioning practitioners, giving them insights into the running performance in the OGK situation and serving as guidelines for training.

\section{Acknowledgements}

The authors would like to thank the Brazilian National Council for Scientific and Technological Development (CNPq, protocol number: 446548/2014-6), Fundação Araucária (protocol number: 25372/2013), and the São Paulo Research Foundation (FAPESP, grant \#2019/17729-0) for the financial support in this research. This study was financed in part by the Coordenação de Aperfeiçoamento de Pessoal de Nível Superior, Brazil (Finance Code: 001).

\section{Disclosure statement}

No author has any financial interest or received any financial benefit from this research.

\section{Conflict of interest}

The authors state no conflict of interest.

\section{References}

1. Barris S, Button C. A review of vision-based motion analysis in sport. Sports Med. 2008;38(12):1025-1043; doi: 10.2165/00007256-200838120-00006.

2. Travassos B, Araújo D, Vilar L, McGarry T. Interpersonal coordination and ball dynamics in futsal (indoor football). Hum Mov Sci. 2011;30(6):1245-1259; doi: 10.1016/j.humov.2011.04.003.

3. Caetano FG, de Oliveira Bueno MJ, Marche AL, Nakamura FY, Cunha SA, Moura FA. Characterization of the sprint and repeated-sprint sequences performed by professional futsal players, according to playing position, during official matches. J Appl Biomech. 2015;31(6): 423-429; doi: 10.1123/jab.2014-0159.

4. Figueroa PJ, Leite NJ, Barros RML. Tracking soccer players aiming their kinematical motion analysis. Comput Vis Image Underst. 2006;101(2):122-135; doi: 10.1016/j.cviu.2005.07.006.

5. Dogramaci SN, Watsford ML, Murphy AJ. Time-motion analysis of international and national level futsal. J Strength Cond Res. 2011;25(3):646-651; doi: 10.1519/JSC.0b013e3181c6a02e.

6. Makaje N, Ruangthai R, Arkarapanthu A, Yoopat P. Physiological demands and activity profiles during futsal match play according to competitive level. J Sports Med Phys Fitness. 2012;52(4):366-374.

7. Barbero-Alvarez JC, Soto VM, Barbero-Alvarez V, Granda-Vera J. Match analysis and heart rate of futsal players during competition. J Sports Sci. 2008;26(1):6373; doi: 10.1080/02640410701287289.

8. Palucci Vieira LH, Doğramaci SN, Barbieri RA, Milioni F, Moura FA, de Andrade VL, et al. Preliminary results on organization on the court, physical and tech- 
nical performance of Brazilian professional futsal players: comparison between friendly pre-season and official match. Motriz. 2016;22(2):79-91; doi: 10.1590/ s1980-6574201600020011.

9. De Oliveira Bueno MJ, Caetano FG, Costa Pereira TJ, de Souza NM, Moreira GD, Nakamura FY, et al. Analysis of the distance covered by Brazilian professional futsal players during official matches. Sports Biomech. 2014;13(3):230-240; doi: 10.1080/14763141.2014.95 8872.

10. FIFA. Futsal. Laws of the game 2014/2015. Zurich: FIFA; 2015.

11. Méndez C, Gómez MA, Rúiz LM, Travassos B. Goalkeeper as an outfield player: shooting chances at critical moments in elite futsal. Int J Perform Anal Sport. 2019;19(2):179-191; doi: 10.1080/24748668.2019.15 81967.

12. Corrêa UC, Davids K, Silva SL, Denardi RA, Tani G. The influence of a goalkeeper as an outfield player on defensive subsystems in futsal. Adv Phys Educ. 2014; 4(2):84-92; doi: 10.4236/ape.2014.42012.

13. Castagna C, D’Ottavio S, Granda Vera J, Barbero Alvarez JC. Match demands of professional futsal: a case study. J Sci Med Sport. 2009;12(4):490-494; doi: 10.1016/j.jsams.2008.02.001.

14. Cohen J. Statistical power analysis for the behavioral sciences. New York: Lawrence Erlbaum Associates; 1988.

15. Gonçalves BV, Figueira BE, Maçãs V, Sampaio J. Effect of player position on movement behaviour, physical and physiological performances during an 11-a-side football game. J Sports Sci. 2014;32(2):191-199; doi: 10.1080/02640414.2013.816761.

16. Moura FA, van Emmerik REA, Santana JE, Barreto Martins LE, Leite de Barros RM, Cunha SA. Coordination analysis of players' distribution in football using cross-correlation and vector coding techniques. J Sports Sci. 2016;34(24):2224-2232; doi: 10.1080/02640414. 2016.1173222 . 